\title{
Power System Stability Improvement Using Dynamic Output Feedback Compensators
}

\author{
CHERN-LIN CHEN and YUAN-YIH HSU \\ Department of Electrical Engineering, National Taiwan University, Taipei (Taiwan) \\ (Received October 3, 1986)
}

\section{SUMMARY}

A novel approach is presented for the design of physically realizable dynamic output feedback compensators for linear time-invariant single-input single-output systems. The simplicity and effectiveness of the proposed method is demonstrated by an example of feedback compensator design for a power system.

\section{INTRODUCTION}

In a recent paper, Lim and Elangovan [1] presented a method for the pole assignment of single-input single-output (SISO) systems using dynamic compensators. The method is effective and is applicable to systems of any order. However, the matrix reduction technique proposed in ref. 1 , which is the kernel of the whole solution procedure, can only be applied to a special class of systems where the compensator input is one of the state variables. To overcome this undesirable limitation, we present an alternative approach for the pole assignment of linear time-invariant single-variate systems. The proposed method can be applied to general SISO systems.

\section{PROPOSED METHOD}

Consider a controllable SISO linear system

$\dot{\boldsymbol{x}}=\mathrm{Ax}+\mathrm{B} u$

$\boldsymbol{y}=\mathbf{C x}$

where $x$ is the $n \times 1$ state vector, $u$ is the scalar input, $y$ is the scalar output, and A, B, and $\mathbf{C}$ are system matrices with dimensions of $n \times n, n \times 1$, and $1 \times n$ respectively. Taking the Laplace transform of (1) and (2), we have the state and output equations in the frequency domain:

$$
\begin{aligned}
& s X(s)=\mathrm{A} X(s)+\mathrm{B} U(s) \\
& Y(s)=\mathrm{C} X(s)
\end{aligned}
$$

Equation (3) can be rearranged as follows:

$$
X(s)=(s \mathbf{I}-\mathbf{A})^{-1} \mathbf{B} U(s)
$$

Let $H(s)$ be the transfer function of the dynamic output feedback compensator, then

$$
U(s)=H(s) Y(s)
$$

Combining eqns. (4), (5), and (6), we have

$X(s)=(s \mathbf{I}-\mathbf{A})^{-1} \mathbf{B} H(s) \mathbf{C X}(s)$

or

$\left[\mathrm{I}-(s \mathrm{I}-\mathrm{A})^{-1} \mathrm{~B} H(s) \mathrm{C}\right] X(s)=0$

If $\lambda$ is the assigned eigenvalue of the closedloop system equipped with the dynamic output feedback compensator, then

$\operatorname{det}\left[\mathbf{I}-(\lambda \mathbf{I}-\mathbf{A})^{-1} \mathbf{B} H(\lambda) \mathbf{C}\right]=0$

Using the identity of determinants [2]

$\operatorname{det}[\mathbf{I}-\mathbf{E} \cdot \mathbf{F}]=\operatorname{det}[\mathbf{I}-\mathbf{F} \cdot \mathbf{E}]$

eqn. (9) can be written as

$$
1-\mathbf{C}(\lambda \mathbf{I}-\mathbf{A})^{-1} \mathbf{B} H(\lambda)=0
$$

or

$$
H(\lambda)=\frac{1}{\mathbf{C}(\lambda \mathbf{I}-\mathbf{A})^{-1} \mathbf{B}}
$$

Equation (12) is the basis for the determination of the compensator parameters. 


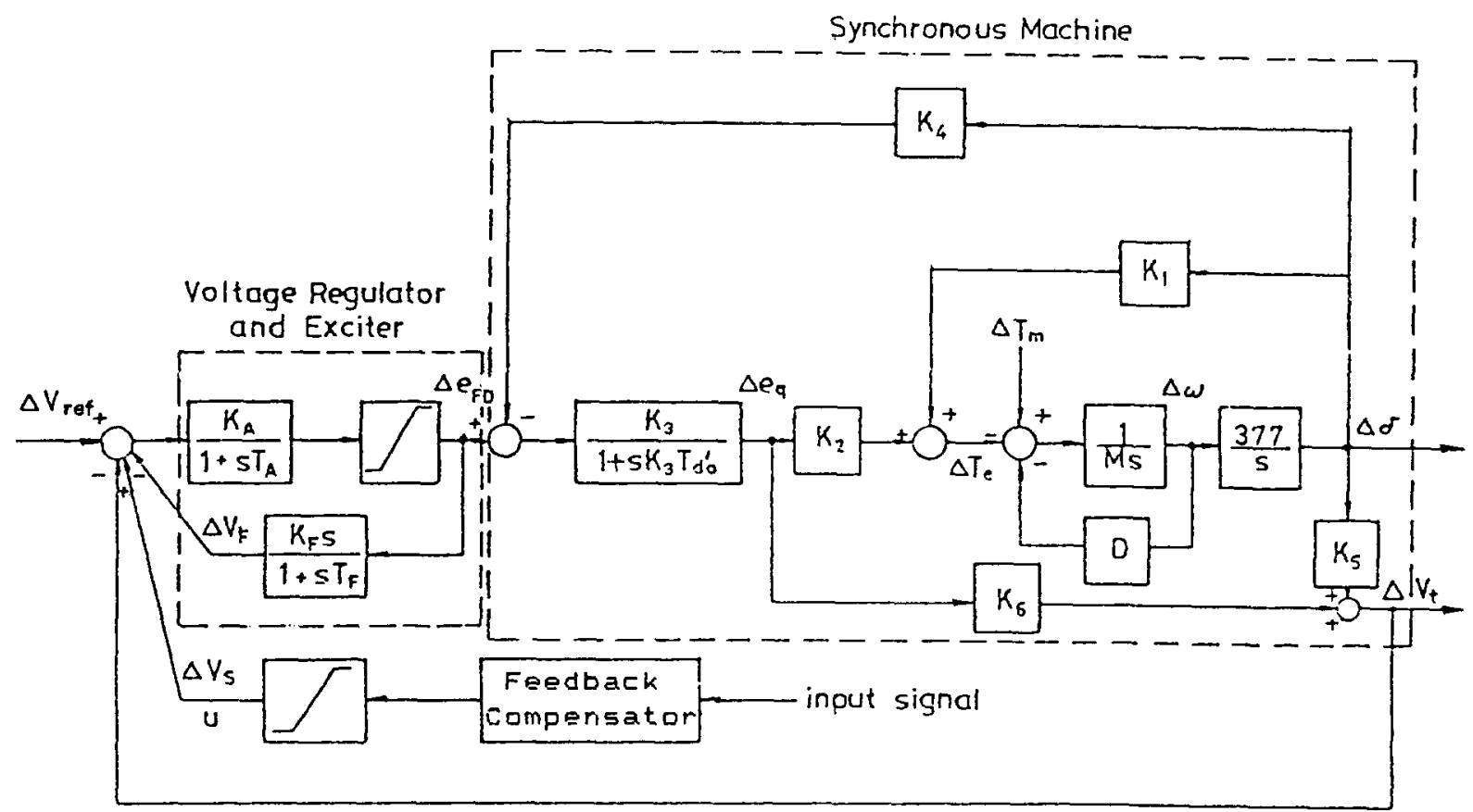

Fig. 1. Linearized incremental model of a synchronous machine equipped with an exciter.

EXAMPLE

Consider a single-machine infinite-bus system whose linearized incremental model including the voltage regulator and exciter can be represented by the block diagram in Fig. 1 [3]. The state equation for a certain operating point can be written in the form

$\dot{X}=\mathbf{A} X+\mathbf{B} U$

where

$X=\left[\begin{array}{lllll}\Delta \delta & \Delta \omega & \Delta e_{\mathrm{q}}^{\prime} & \Delta e_{\mathrm{FD}} & \Delta v_{\mathrm{f}}\end{array}\right]^{\mathrm{T}}$

$\mathbf{A}=\left[\begin{array}{clllc}0 & 377 & 0 & 0 & 0 \\ -0.227 & 0 & -0.265 & 0 & 0 \\ -0.29 & 0 & -0.552 & 0.169 & 0 \\ 327.2 & 0 & -3976.8 & -20 & -8000 \\ 8.18 & 0 & -99.42 & -0.5 & -201\end{array}\right]$

$B=\left[\begin{array}{llllll}0 & 0 & 0 & 8000 & 200\end{array}\right]^{\mathrm{T}}$

The eigenvalues of the original open-loop system are shown in the first column of Table 1. Now a proportional-integral (PI) compensator with a washout term is to be installed in order to improve system damping [4]. The transfer function of the compensator is
$H(s)=\frac{s}{1+s}\left(K_{\mathrm{p}}+\frac{K_{i}}{s}\right)$

If $\Delta \omega$ is chosen as the compensator input, then

$\mathbf{C}=\left[\begin{array}{lllll}0 & 1 & 0 & 0 & 0\end{array}\right]$

If $\Delta T_{\mathrm{e}}$ is the input of the compensator, then

$\mathrm{C}=\left[\begin{array}{lllll}1.0755 & 0 & 1.2578 & 0 & 0\end{array}\right]$

Using eqn. (12) and assuming $\lambda$ and $\lambda^{*}$ are the desired system eigenvalues,

$\frac{\lambda}{1+\lambda}\left(K_{\mathrm{p}}+\frac{K_{\mathrm{i}}}{\lambda}\right)=\frac{1}{\mathrm{C}(\lambda \mathrm{I}-\mathbf{A})^{-1} \mathbf{B}}$

$K_{\mathrm{p}}$ and $K_{\mathrm{i}}$ can be derived as follows:

$K_{\mathfrak{p}}=\frac{\operatorname{Im}\left\{(1+\lambda) /\left[\mathbf{C}(\lambda \mathbf{I}-\mathbf{A})^{-1} \mathbf{B}\right]\right\}}{\operatorname{Im}\{\lambda\}}$

$K_{i}=\operatorname{Re}\left\{\frac{1+\lambda}{\mathbf{C}(\lambda \mathbf{I}-\mathbf{A})^{-1} \mathbf{B}}\right\}-K_{p} \times \operatorname{Re}\{\lambda\}$

Using these formulas, the compensator parameters $K_{\mathrm{p}}$ and $K_{\mathrm{i}}$, and the corresponding closed-loop system eigenvalues for the cases of $\Delta \omega$ feedback and $\Delta T_{\mathrm{e}}$ feedback are computed and are given in the second and third columns of Table 1 , respectively. It is found 


\section{TABLE 1}

System eigenvalues with and without compensator

\begin{tabular}{llll}
\hline & Without compensator & \multicolumn{2}{l}{ With compensator } \\
\cline { 3 - 4 } & & $\Delta \omega$ feedback & $\Delta T_{\mathrm{e}}$ feedback \\
\hline$K_{\mathrm{p}}$ & - & 1.981 & -0.193 \\
$K_{\mathrm{i}}$ & - & -78.202 & -0.727 \\
System eigenvalues & -217.8 & -217.8 & -216.3 \\
& $-0.0138 \pm \mathrm{j} 9.222$ & $-0.8 \pm \mathrm{j} 9.222^{\mathrm{a}}$ & $-0.8 \pm \mathrm{j} 9.222^{\mathrm{a}}$ \\
& $-1.852 \pm \mathrm{j} 0.0382$ & $-1.053 \pm \mathrm{j} 1.513$ & $-1.817 \pm \mathrm{j} 0.359$ \\
& & -1.0 & -1.0 \\
\hline
\end{tabular}

a Exactly assigned eigenvalues.

that the prespecified eigenvalues are assigned exactly by the proposed approach, no matter what the input signal of the compensator is.

\section{CONCLUSIONS}

A simple method has been proposed for the pole assignment of single-variate systems using dynamic output compensators. The main feature of the proposed approach is its capability to design a compensator whose input signal is not one of the state variables. The effectiveness of the proposed method has been demonstrated by the design of a com- pensator with different input signals for an electric power system.

\section{REFERENCES}

1 C. M. Lim and S. Elangovan, New approach to power system stabilizer design, Electr. Power Syst. Res., 8 (1985) 285 - 292.

2 T. Kailath, Linear Systems, Prentice Hall, Englewood Cliffs, NJ, 1980, p. 651.

3 F. P. deMello and C. Concordia, Concepts of synchronous machine stability as affected by excitation control, IEEE Trans., PAS-88 (1969) $316-329$.

$4 \mathrm{Y}$. Y. Hsu and C. Y. Hsu, Design of a proportional-integral power system stabilizer, IEEE Trans., PWRS-1 (1986) 46 - 53. 